\title{
Some Features of Interphase Phenomena in Heat-Conducting Composite Materials Used in Microelectronics
}

\author{
H.S. Aliyev \\ Azerbaijan Technical University, H.Javid ave 25, Baku, Azerbaijan AZ 1073
}

*Corresponding Author: H.S. Aliyev, Azerbaijan Technical University, H.Javid ave 25, Baku, Azerbaijan AZ 1073

\begin{abstract}
In our experiments, we found that the coefficients $\lambda$ of thermal conductivity of composites based on low density polyethylene (LDPE) and various nitrides and carbides (aluminum nitride AlN, boron nitride BN, titanium nitride TiN, etc.) differ markedly. A more efficient heat-conducting composite among the considered composites is the LDPE $+B N$ composite. Such a difference in the $\lambda$ values of these composites indicates the role of interfacial interactions between the dispersed medium (polymer) and the dispersant (metal nitridescarbides). Of particular importance here, in our opinion, are processes at the phase interface, as well as the effect of the surface states of dispersant particles on the physical and chemical structure of the polymer matrix.
\end{abstract}

Keywords: polymer, dispersant, composite, matrix, structure, thermal conductivity.

\section{INTRODUCTION}

The dispersion of the polymer by an inorganic particle is accompanied by the formation of new elements of the supermolecular structure (SMS) under the influence of the particle surface, and the larger the volumetric content of the inorganic phase, the more part of the polymer volume is formed under the influence of the dispersant. With the complete transition of the polymer phase to the nearsurface layer with an increase in the volume content of the dispersant, the structure of the polymer throughout the volume is formed under the influence of the particle surface. Therefore, depending on the activity or surface state of the dispersant particles, the heterogeneity of the physical and chemical structures of the polymer phase will differ, and this, in our opinion, will determine the thermophysical properties of the composite polymer-nitrides, carbides-metals [1].

We believe that the heat-conducting properties are mainly determined by the ratio of the scattered and transferred thermal energies across the interface. Therefore, in these processes, the mobility of the kinetic units of macromolecules in the surface layers, the free volume at the interface, as well as possible chemical bonds between the surface atoms of the dispersant particles and macromolecules are of great importance. The ratio of thermal conductivity of fillers and composites based on them is not equal, which indicates the decisive role of the heterogeneity of the polymer phase between particles.

\section{EXPERIMENT}

Experiments show that in order to develop more efficient heat-conducting composites, it is necessary to predict and purposefully regulate processes at the interface of the composite phases. The differences in the values of thermal conductivity $(\lambda)$ of these composites are shown in Figure 1.

The graphs indicate the effect of the density structure and surface states of the filler particles on the physical and chemical structures of the polymer phase, on which the thermal conductivity of the composite strongly depends. In our opinion, a noticeable increase in $\lambda$ after $\Phi$ of more than $30 \mathrm{wt} \%$ is associated with the fact that at such values of $\Phi$ the effect of the formation of the structure of the polymer phase under the influence of the surface of the filler particles increases [2,3].

International Journal of Innovative Research in Electronics and Communications (IJIREC) Page | 1 
This shows that the supramolecular structure of the polymer phase and the mobility of the kinetic units of macromolecules, including segments at the interface, determine the thermal conductivity of multiphase systems.

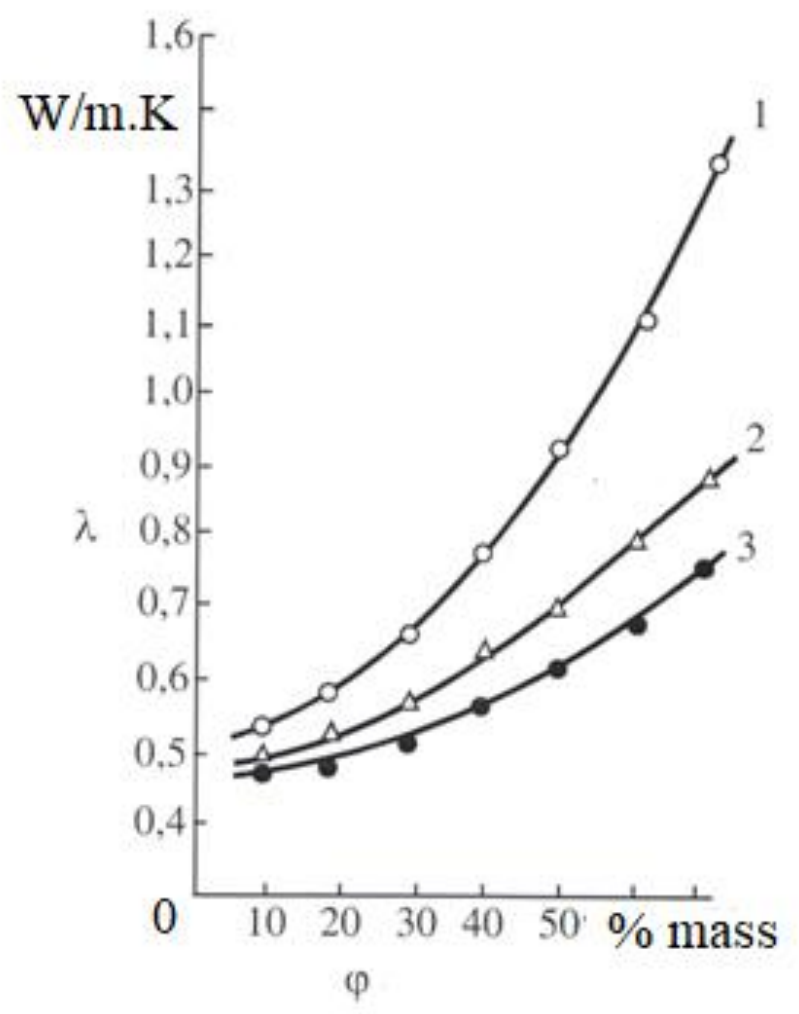

Fig1. Dependence of thermal conductivity $\lambda$ of low density polyethylene (LDPE) on the content of $F$ nitrides and metal carbides. 1 - $B N ; 2$ - $\mathrm{Ti}_{3} \mathrm{~N}_{4} ; 3$ - TiCN.

Interphase and intermolecular phenomena are capable of changing the physicomechanical and thermophysical properties of the polymer matrix in heterogeneous composite systems. The degree of these interactions will determine the heterogeneity of the structures, the degree of crystallinity and adhesion of the polymer phase to the surface of the filler particles and the development of a chemical reaction at the interface. These factors, in turn, will affect the thermal conductivity of composites. The degree of interaction between the polymer and the filler, as well as between the macromolecules of the polymer phase, can be judged by studying the thermophysical and thermoelectric properties of composites. For the first time, we used the posistor effect in composites to predict their thermal conductivity [4].

Figure 2 shows the temperature dependences of the resistivity of composites based on high-density polyethylene (HDPE) with fillers (BN, AIN) with a filler volume content $\Phi=30 \%$ vol. It can be seen that a posistor effect is clearly observed in these composites, that is, they are thermistors with a positive temperature resistance.

As can be seen from Figure 2, the position of the minimum electrical conductivity depends on the type of filler. In the case of the HDPE + BN composite, the temperature of the minimum of the dependence $\ln \sigma=\mathrm{f}(1 / \mathrm{T})$ is higher than for the HDPE + AlN composite, which indicates a relatively high interfacial interaction. The temperature dependence of the electrical conductivity of composites can be explained based on the tunneling mechanism of charge transfer between particles in composites [5,6]. The polymer-filler composite system consists of two phases that differ sharply in their electrophysical properties, which leads to the formation of barriers at the phase interface. 


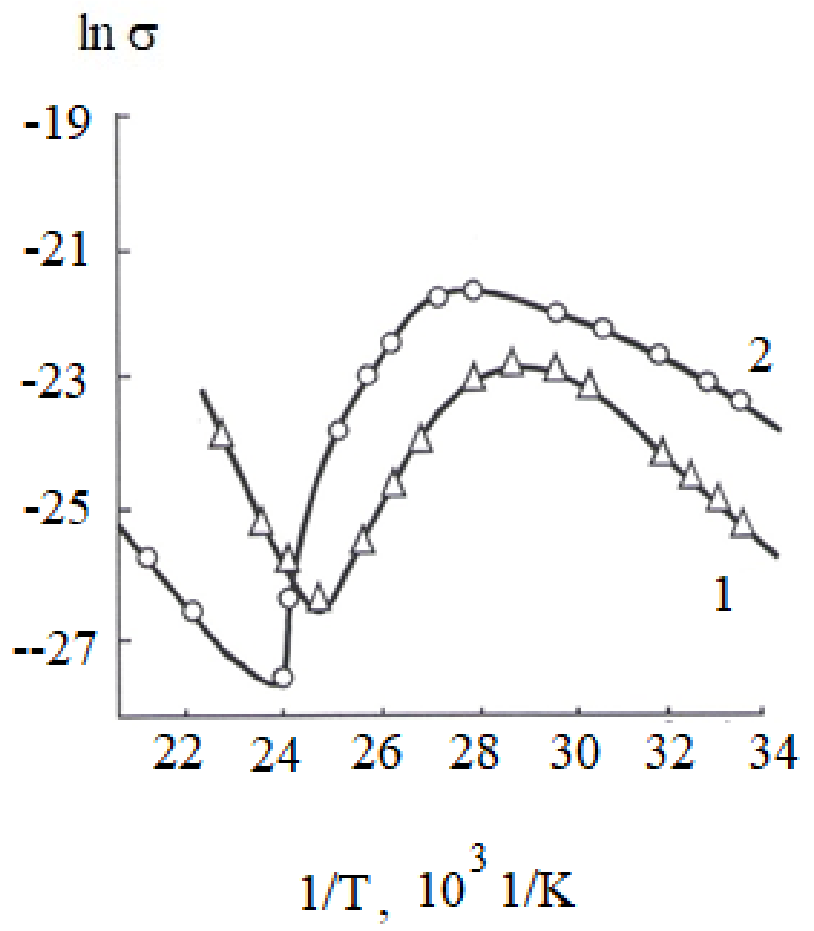

Fig2. Dependences of electrical conductivit ln $\sigma$ on 1/T: $1-H D P E+B N ; 2-H D P E+A l N$.

At certain temperatures, the contribution of the thermal expansion of the polymer matrix becomes significant and the value of the boundary barrier increases, and, therefore, the increase in electrical conductivity is replaced by a decrease. Finally, when the temperature reaches the melting point of the crystalline phase of the polymer, the volume of the polymer matrix increases sharply, and this leads to an increase in the average distance between the filler particles and, consequently, in the barrier value. The termination of particle tunneling is accompanied by a sharp decrease in the electrical conductivity of the composite. The melting point of the crystalline phase and the half-width of the posistor peak depend on the superamolecular structure of the polymer phase and interphase interaction. Therefore, the dependence $\ln \sigma=\mathrm{f}(1 / \mathrm{T})$ can also be used to predict the thermal conductivity of composites. Let us consider the influence of the pressing time on the posistor effect in heat-conducting composites in order to predict their properties.

\section{THE DISCUSSION OF THE RESULTS}

Figure 3 shows the dependence of the electrical conductivity of the HDPE + BN composite at room temperature $\sigma_{0}$, at the melting point of the crystalline phase $\sigma_{\mathrm{rmin}}$, as well as $\ln \left(\sigma_{1} / \sigma_{\mathrm{rmin}}\right)$ on the pressing time $t_{\mathrm{pr}}$. Here $\sigma_{1}$-is the electrical conductivity of the sample at a temperature corresponding to the beginning of a sharp decrease in electrical conductivity. The value $\ln \left(\sigma_{1} / \sigma_{\mathrm{kmin}}\right)$ characterizes the jump in the electrical conductivity of composites upon melting of the crystalline phase of the polymer matrix and is associated, other things being equal, with the supramolecular structure, in particular, with the degree of crystallinity. It also shows the change in thermal conductivity from the time of pressing (curve - 4), that is, $\lambda=\mathrm{f}\left(\mathrm{t}_{\mathrm{pr}}\right)$. It is seen that the dependence $\lambda=\mathrm{f}\left(\mathrm{t}_{\mathrm{pr}}\right)$ is expressed by a curve with a maximum.

It is clear that $\sigma_{0}$ increases up to $t_{\mathrm{pr}}=10$ minutes and then decreases, at the same time $\sigma_{\mathrm{kmin}}$ changes in the opposite way, and $\sigma_{1} / \sigma_{\text {кmin }}$ changes with tpr in the same way as $\sigma_{0}$. This dependence of the electrical conductivity on tpr can be explained by the fact that with an increase in the pressing time, the degree of crystallinity of the polymer in the composition increases. Crystallites are evenly distributed over the entire volume of the polymer phase and partially expel filler particles from the crystalline phase to the amorphous one. With a further increase in the pressing time tpr, the process of formation of higher elements of the supramolecular structure, in particular, spherulites, occurs. 


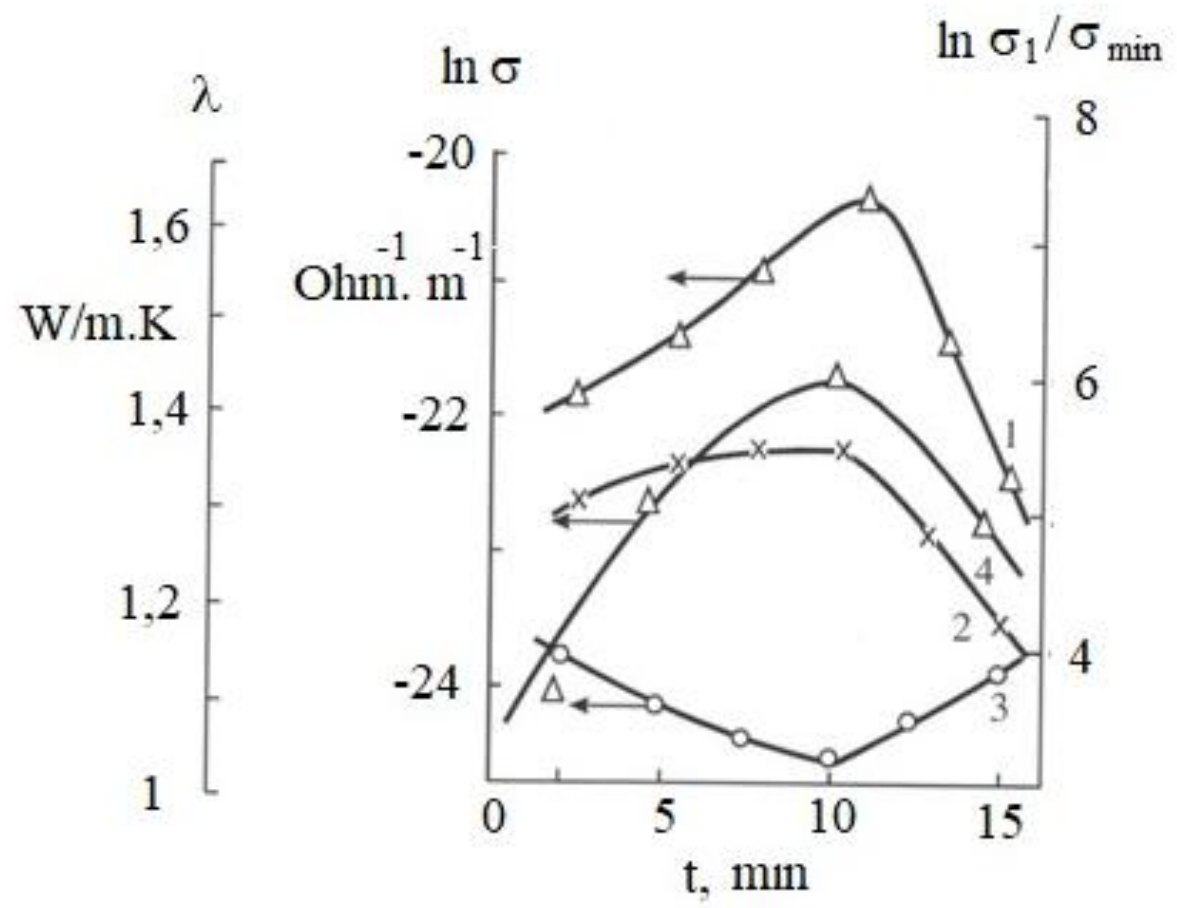

Fig3. $\ln \sigma_{0}, \ln \sigma_{\text {kmin }}$ and $\ln \left(\sigma_{l} / \sigma_{\text {kmin }}\right)$ om $t_{p r} .1-\ln \sigma_{0} ; 2-\left(\ln \sigma_{l} / \sigma_{\kappa m i n}\right) ; 3-\ln \sigma_{\kappa m i n} ; 4-\lambda=f\left(t_{p r}\right)$.

There are new boundaries - interspherolite - except for the boundaries filler - polymer phase. New boundaries are additional hotbeds of heat dissipation, and therefore, the thermal conductivity of the composite decreases as a whole.

Note also that as spherulites are formed, polymer chains are extended from the amorphous region into spherulite, as a result of which the free volume at the spherulite boundary grows. This, apparently, is the main reason for the decrease in the electrical conductivity of the composite.

\section{CONClusions}

Summarizing the results of the study on the posistor effect, which makes it possible to judge the interfacial phenomena in composites based on polyolefins dispersed with $\mathrm{BN}$ or AlN particles, the following points can be highlighted:

1. The change in the temperature of occurrence and the half-width of the posistor peak is determined by interphase interactions.

2. The temperature corresponding to the onset of melting of the crystalline phase depends on the type of storage ring, i.e. from the activity of the particle surface.

3. The transition from the first section to the second in the dependence $\ln \gamma_{\mathrm{V}}=(1 / \mathrm{T})$ occurs smoothly, and the temperature range of this transition depends on the technological conditions of production and the activity of the filler surface.

\section{REFERENCES}

[1] Tuncer E., Gubanski S.M., Nettelblad B. Dielectric relaxation in dielectric mixtures: Application of the finite element method and its comparison with dielectric mixture formulas // Journal of Applied Physics.2001.- Vol. 89. № 12.- P. 8092- 8100.

[2] Sihvola A. Mixing rules with complex dielectric coefficients // Subsurface Sensing Technologies and Applications.- 2000.- Vol. 1. № 4. P. 393- 414.

[3] Bai Y., Cheng Z.-Y., Bharti V., Xu H. S., Zhang Q. M. High-dielectric-constant ceramic-powder polymer composites // Applied Physics Letters. -2000.- Vol. 76. № 25.- P. 3804- 3806.

[4] Tchmyreva V.V., Ponomarenko A.T., Shevchenko V.G. Structure and dielectric properties of polymeric composites with ferroelectric fillers // e-Polymers, www.e-polymers.org.- 2003. № 036.- P. 1- 12. 
[5] Steeman P. A.M., Baetsen J.F.H., Maurer F.H.J. Temperature dependence of the interfacial dielectric loss process in glass bead filled polyethylene // Polymer Engineering and Science.- 1992.- Vol. 32. № 5.- P. 351- 356.

[6] Jayasundere N., Smith B.V., Dunn J.R. Piezoelectric constant for binary piezoelectric 0-3 connectivity composites and the effect of mixed connectivity //Journal of Applied Physics.- 1994.- Vol. 76. № 5.- P. 2993- 2998.

Citation: H.S. Aliyev, (2020)" Some Features of Interphase Phenomena in Heat-Conducting Composite Materials Used in Microelectronics", International Journal of Innovative Research in Electronics and Communications (IJIREC), 7(3), pp. 1-5. DOI: http://doi.org/10.20431/2349-4050.0703001

Copyright: (C) 2020 H.S. Aliyev, This is an open-access article distributed under the terms of the Creative Commons Attribution License, which permits unrestricted use, distribution, and reproduction in any medium, provided the original author and source are credited. 